Groups Geom. Dyn. 4 (2010), 1-13

DOI $10.4171 / \mathrm{GGD} / 72$
Groups, Geometry, and Dynamics

(C) European Mathematical Society

\title{
Geometric characterization of flat groups of automorphisms
}

\author{
Udo Baumgartner, Günter Schlichting and George Willis*
}

\begin{abstract}
If $\mathscr{H}$ is a flat group of automorphisms of finite rank $n$ of a totally disconnected, locally compact group $G$, then each orbit of $\mathscr{H}$ in the metric space $\mathscr{B}(G)$ of compact, open subgroups of $G$ is quasi-isometric to $n$-dimensional Euclidean space. In this note we prove the following partial converse: Assume that $G$ is a totally disconnected, locally compact group such that $\mathscr{B}(G)$ is a proper metric space and let $\mathscr{H}$ be a group of automorphisms of $G$ such that some (equivalently every) orbit of $\mathscr{H}$ in $\mathscr{B}(G)$ is quasi-isometric to $n$-dimensional Euclidean space, then $\mathscr{H}$ has a finite index subgroup which is flat of rank $n$. We can draw this conclusion under weaker assumptions. We also single out a naturally defined flat subgroup of such groups of automorphisms.
\end{abstract}

Mathematics Subject Classification (2010). 22D05, 22D45, 20E25, 20E36.

Keywords. Totally disconnected locally compact group, automorphism group, tidy subgroup, rank, quasi-isometry, flat.

\section{Introduction}

In this article all automorphisms of topological groups are assumed to be continuous with continuous inverse. Flat groups of automorphisms of a totally disconnected, locally compact group were introduced and studied in [Wil04]. A group of automorphisms, $\mathscr{H}$ say, of a totally disconnected, locally compact group $G$ is flat if there exists a compact, open subgroup, $O$ say, of $G$, called minimizing for $\mathscr{H}$, that minimizes all the displacement functions of elements in $\mathscr{H}$ on the metric space, $(\mathscr{B}(G), d)$, of compact, open subgroups of $G$ relative to the distance function $d(V, W):=\log (|V: V \cap W| \cdot|W: W \cap V|)$. One can rephrase this condition by saying that for every $\varphi \in \mathscr{H}$ the integer $|\varphi(O): \varphi(O) \cap O|$ attains the value of the scale function, $s_{G}$, at $\varphi$, where $s_{G}$ is defined by $s_{G}(\varphi):=\min \{|\varphi(V): \varphi(V) \cap V|: V \in$ $\mathscr{B}(G)\}$.

Flat groups are, in a sense, generalizations of split tori in semisimple algebraic groups over nonarchimedian local fields. They admit a dynamically defined 'root system' $\Phi_{\mathscr{H}}$, which governs the decomposition of any minimizing subgroup for $\mathscr{H}$

\footnotetext{
*Authors were supported by Australian Research Council grant DP0556017.
} 
into a product of associated 'eigenfactors' on which every element of $\mathscr{H}$ is either expanding or contracting.

In this article we study the question of the extent to which flat groups are characterized by their geometric properties. These properties are summarized in the following theorem.

Theorem 1.1 (Geometric properties of flat groups of automorphisms). Let $\mathscr{H}$ be $a$ flat group of automorphisms of a totally disconnected, locally compact group $G$ and let $O$ be minimizing for $\mathscr{H}$. Put $\mathscr{H}(1):=\left\{\varphi \in \mathscr{H}: s_{G}(\varphi)=1=s_{G}\left(\varphi^{-1}\right)\right\}$. Then

(1) $\mathscr{H}_{O}=\mathscr{H}(1) \unlhd \mathscr{H}$ and $\mathscr{H} / \mathscr{H}(1)$ is a free abelian group, whose $\mathbb{Z}$-rank is called the rank of $\mathscr{H}$;

(2) there is a set $\Phi_{\mathscr{H}} \subseteq \operatorname{Hom}(\mathscr{H}, \mathbb{Z})$ of surjective homomorphisms (which is independent of $O)$ such that $\bigcap_{\rho \in \Phi_{\mathcal{H}}} \operatorname{ker}(\rho)=\mathscr{H}(1)$ and such that for each $\varphi \in \mathscr{H}$ we have $\rho(\varphi) \neq 0$ for only finitely many $\rho$ in $\Phi_{\mathscr{H}}$;

(3) there are positive integers $t_{\rho}$ for $\rho \in \Phi_{\mathcal{H}}$ (which are independent of $O$ ) such that the function $\|\cdot\|_{\mathscr{H}}$ defined on $\mathscr{H} / \mathscr{H}(1)$ by the rule $\left.\| \varphi \mathscr{H}(1)\right) \|_{\mathscr{H}}:=d(\varphi(O), O)$ takes the value $\sum_{\rho \in \Phi_{\mathcal{H}}} \log \left(t_{\rho}\right)|\rho(\varphi)|$ at $\varphi \mathscr{H}(1)$ for $\varphi \in \mathscr{H}$.

Hence the function $\|\cdot\|_{\mathscr{H}}$ extends to a norm on the vector space $\mathbb{R} \otimes \mathscr{H} / \mathscr{H}(1)$ and each orbit of a flat group of automorphisms of finite rank in $\mathcal{B}(G)$ is quasi-isometric to $\mathbb{R} \otimes \mathscr{H} / \mathscr{H}(1)$.

Theorem 1.1 follows from results proven in the paper [Wil04]; the reader may consult the survey article [Bau07] for a compact exposition, with only short hints of proofs; the statement on all orbits of $\mathscr{H}$ in $\mathcal{B}(G)$ is obtained by applying the following lemma with $\boldsymbol{B}:=\mathscr{B}(G)$ and the natural action of $\mathscr{H}$ on $\boldsymbol{B}$. The proof of the lemma is left to the reader.

Lemma 1.2 (Distances on orbits under isometric actions differ by a constant). Let a group $\mathscr{H}$ act by isometries on a metric space $\boldsymbol{B}$. Then any two orbits of $\mathscr{H}$ in $\boldsymbol{B}$ are $(1, \varepsilon)$-quasi-isometric, with $\varepsilon$ only depending on the pair of orbits. In particular all orbits of $\mathscr{H}$ in $\boldsymbol{B}$ have the same type of growth.

In this paper we address the question whether flat groups are virtually characterized by the rough isometry class of their orbits in the space of compact, open subgroups; more precisely, we study the following problem.

Problem 1.3 (Question whether flatness can be characterized geometrically). Let $G$ be a totally disconnected, locally compact group and let $\mathscr{H}$ be a group of automorphisms of $G$ such that some (equivalently, any) orbit of $\mathscr{H}$ in the metric space of compact, open subgroups of $G$ is quasi-isometric to $\mathbb{R}^{n}$ for some $n$ in $\mathbb{N}$. Does it follow that $\mathscr{H}$ has a finite index subgroup which is a flat group of automorphisms of $G$ of rank $n$ ? 
Under the conditions described in Problem 1.3, the group $\mathscr{H}$ itself need not be flat; two examples illustrating this will be given in Section 5.

A geometric characterization as formulated in Problem 1.3 can be expected to be useful in the detection and classification of flat subgroups of totally disconnected, locally compact groups that are automorphism groups of a geometric structure because for such groups the space of compact, open subgroups will often be related to that geometric structure. An example is presented in Theorem 5.3.

While we can not solve Problem 1.3 in full generality, Theorem 1.4 below solves it affirmatively in the important case where the metric space of compact, open subgroups is proper; we are actually able to obtain the same conclusion as in Theorem 1.4 under weaker hypotheses, see Theorem 2.3.

We also have quite strong control over the flat group of finite index whose existence we guarantee; compare Theorem 2.5. However, there is probably no way to describe a minimizing subgroup for that flat subgroup of finite index.

Abstract flatness criteria that do not effectively produce a minimizing subgroup have been proved before: a purely algebraic criterion by Willis in [Wi104] and several 'algebraic-bounded' criteria by Shalom and Willis in [SW07], one of which, restated here as Theorem 2.2, we use in the proof of Theorems 1.4 and 2.3.

Theorem 1.4 (Geometric characterization of flatness in the proper case). Let $G$ be a totally disconnected, locally compact group and let $\mathscr{H}$ be a group of automorphisms of $G$ such that some (equivalently, any) orbit of $\mathcal{H}$ in the metric space of compact, open subgroups of $G$ is quasi-isometric to $\mathbb{R}^{n}$ for some $n$ in $\mathbb{N}$. Assume that the metric space $\mathcal{B}(G)$ of compact, open subgroups of $G$ is proper. Then $\mathscr{H}$ has a finite index subgroup which is a flat group of automorphisms of $G$ of rank $n$.

We now discuss known results that are similar to and, it turns out, also related to Theorem 1.4. These results illustrate why one might expect to obtain such a result in the first place.

If we ignore the special form of the group $\mathscr{H}$ that acts on the quasi-flat orbits, what we seek may be called a 'quasi-isometric version' of Bieberbach's First Theorem on space groups. (A statement of Bieberbach's First Theorem can be found e.g. in [Aus65], Bieberbach Theorem 1).

We are not aware of the existence of such a 'quasi-isometric version' of Bieberbach's First Theorem, but several well-known results have similar conclusions. For example, by Théorème 17 in [GdlH90], $\mathbb{Z}^{n}$ is quasi-isometrically rigid for every $n$, that is, a finitely generated group that is quasi-isometric to a free abelian group of rank $n$ has a subgroup of finite index that is a free abelian group of rank $n$. The latter result relied until Shalom's paper [Sha04] (Theorem 1.1, proven on p. 126; see also Corollary 1.5 in [dCTV07], proven in §4.3) on Gromov's characterization of finitely generated groups of polynomial growth, which will also play a prominent role in this paper. 
A graph-theoretic analogue of Bieberbach's First Theorem may be seen in Theorem 1 of Trofimov's paper [Tro84] (cited as Theorem 2.1 in the survey paper [IS91]). Trofimov's work [Tro84] implies Bieberbach's First and Third Theorem as noted on p. 417 of that article and also implies Gromov's characterization of finitely generated groups of polynomial growth (but note that the main result in the paper [Gro81] that shows Gromov's result is used in Trofimov's work). In the graph-theoretic context the papers [LS01], [Sei91] also discuss interesting aspects. The above examples and our own success in proving an analogue of Bieberbach's First Theorem in our, admittedly restricted, context suggest that it might be possible and worthwhile to prove a 'quasi-isometric version' of Bieberbach's Theorems on space groups.

It would also be of interest to determine whether our Main Theorem can alternatively be derived using Theorem 1 in Trofimov's paper [Tro84], thus avoiding the use of Losert's result [Los01], Proposition 1 (Losert's result is used on p. 6 in the middle of the proof of Theorem 2.1).

\section{Outline of the proof of the Main Theorem and statement of further results}

As our first step, we use a structure result on compactly generated, locally compact groups of polynomial growth due to Losert together with Gromov's theorem on finitely generated groups of polynomial growth to show Theorem 2.1 below.

We now recall terminology used in the statement of that theorem. Let $d>0$. A metric space is called $d$-connected if and only if for any ordered pair $(x, y)$ of points in the space one can find a finite sequence of points beginning with $x$ and ending with $y$, whose consecutive terms are at most $d$ apart. A metric space is called coarsely connected if and only if it is $d$-connected for some positive number $d$. The image of a coarsely connected space under a quasi-isometric embedding as well as any $\varepsilon$-neighborhood of a coarsely connected space is itself coarsely connected; in particular, the property of being coarsely connected is invariant under quasi-isometry. Further, call a set of automorphisms $B$ of a totally disconnected, locally compact group $G$ bounded if and only if the set $B . V$ has bounded diameter for some (equivalently, every) $V$ in $\mathcal{B}(G)$.

If in Theorem 2.1 the group $\mathscr{H}$ is flat of finite rank, then any minimizing subgroup for $\mathscr{H}$ satisfies the conditions on the group $O$ in that theorem by part 3 of Theorem 1.1. Later in the paper we will use this observation. In Section 5, we will give examples of groups of automorphisms whose orbits are quasi-flats which are not flat.

Theorem 2.1 (Automorphism groups with a proper, coarsely connected orbit of polynomial growth are virtually bounded-by-finitely-generated-nilpotent). Let $G$ be a totally disconnected, locally compact group and $\mathscr{H} \leqslant \operatorname{Aut}(G)$. Assume that there is an $O$ in $\mathcal{B}(G)$ such that the orbit $\mathscr{H} . O$ is proper, coarsely connected and of polynomial growth. Then there is a subgroup $\mathscr{H}_{0}$ of finite index in $\mathscr{H}, \mathcal{N}_{0} \unlhd \mathscr{H}_{0}$ and $V \in \mathscr{B}(G)$ such that $\mathcal{N}_{0}$ stabilizes $V$ and $\mathscr{H}_{0} / \mathcal{N}_{0}$ is a finitely generated nilpotent group. 
We then apply a flatness criterion from [SW07], restated here as Theorem 2.2.

Theorem 2.2 (Bounded-by-finitely-generated-nilpotent groups are flat). Let $\mathcal{N}_{0} \unlhd$ $\mathscr{H}_{0} \leqslant \operatorname{Aut}(G)$ and suppose that $\mathcal{N}_{0}$ stabilizes some compact, open subgroup $V$ of $G$ and that $\mathscr{H}_{0} / \mathcal{N}_{0}$ is a finitely generated nilpotent group. Then $\mathscr{H}_{0}$ is flat of finite rank.

Combining Theorems 2.1 and 2.2 we conclude that the subgroup $\mathscr{H}_{0}$ found in Theorem 2.1 is flat. Thus, we derive the following theorem, modulo the claim contained therein on the rank of $\mathscr{H}_{0}$. That theorem delivers the same conclusion as our desired result from weaker hypotheses and will thus prove our Main Theorem, Theorem 1.4.

Theorem 2.3 (Geometric characterization of flatness if there is a proper orbit). Let $G$ be a totally disconnected, locally compact group and $\mathscr{H} \leqslant \operatorname{Aut}(G)$. Assume that there is an $O$ in $\mathcal{B}(G)$ such that the orbit $\mathscr{H} . O$ is proper, coarsely connected and of polynomial growth of degree $n$ but not $n-1$. Then $\mathscr{H}$ has a subgroup $\mathscr{H}_{0}$ of finite index that is flat of rank $n$.

The strong form of our main theorem, Theorem 2.3, and the remark in the paragraph preceding Theorem 2.1 imply the following corollary.

Corollary 2.4. Let $G$ be a totally disconnected, locally compact group. A group of automorphisms of $G$ whose orbits in $\mathcal{B}(G)$ are quasi-flats of finite dimension is virtually flat if and only if it has a proper orbit in $\mathcal{B}(G)$.

The proof of the existence of the flat subgroup $\mathscr{H}_{0}$ in Theorem 2.3 is not constructive. Nevertheless, $\mathscr{H}$ has a maximal normal flat subgroup, whose elements are characterized intrinsically in the next theorem.

Theorem 2.5. Suppose that $\mathscr{H}$ is a group of automorphisms of a totally disconnected, locally compact group that is virtually flat of finite rank. Then the subset of bounded conjugacy classes of $\mathscr{H}$, defined by $\mathscr{H}_{\mathrm{FC}_{d}}:=\left\{\varphi \in \mathscr{H}: \varphi^{\mathscr{H}}\right.$ is bounded $\}$ is a flat, normal subgroup of $\mathscr{H}$ of finite index that contains each flat subgroup of finite index.

\section{Proof of the geometric flatness criterion if there is a proper orbit}

We follow the outline of the proof given in the last section.

Proof of Theorem 2.1. In the first step of the proof, the assumptions on the orbit $\mathscr{H} . O$ are used to define a locally compact completion, $H$, of $\mathscr{H}$.

Choose $d>0$ such that $\mathscr{H} . O$ is $d$-connected and let $X$ be the metric graph whose set of vertices is $\mathscr{H} . O$ and whose edges connect precisely those pairs of points in $\mathscr{H} . O$ whose mutual distance is at most $d$. The choice of $d$ guarantees that the 
graph $X$ is connected; since $\mathscr{H} . O$ is proper $X$ is also locally finite. The group $\mathscr{H}$ acts by graph-automorphisms on $X$ and the induced action on the set of vertices is transitive.

Denote by $H$ the closure of the group of automorphisms of $X$ induced by $\mathscr{H}$ in the group of all graph-automorphisms of $X$. Since the graph $X$ is connected and locally finite, the group $\operatorname{Aut}(X)$ and its closed subgroup $H$ are totally disconnected and locally compact in the topology of pointwise convergence; indeed, the stabilizer of any vertex is a compact, open subgroup, which is profinite.

Furthermore, because both $\mathscr{H}$ and $H$ act transitively on the set of vertices of $X$, these groups are generated by their respective subsets, $\mathscr{H}_{E}$ respectively $H_{E}$, consisting of those automorphisms that map $O$ to any of its neighbors in $X$. Now the set of elements that map $O$ to another vertex, $O^{\prime}$ say, is a left coset of the stabilizer of $O$; within the group $H$, the latter subset is compact. Since $H_{E}$ is a finite union of such sets, we conclude that it is compact also. Hence the group $H$ is totally disconnected, locally compact and compactly generated.

We claim next that $H$ has polynomial growth. This is seen as follows. The growth of $H$ with respect to $H_{E}$ equals the growth of the combinatorial graph $X$. For any radius, $r$ say, the ball of radius $r$ around $O$ in $\mathscr{H} . O$ with respect to the metric of $\mathcal{B}(G)$ contains the ball with radius $r / d$ with respect to the metric of $X$. Since the former balls grow polynomially, the latter do also and thus $H$ has polynomial growth.

Since $H$ has been seen to be a compactly generated, totally disconnected, locally compact group of polynomial growth, we may apply [Los01], Proposition 1, to $H$ to conclude that it has a maximal compact normal subgroup $C$ such that $H / C$ is a Lie group. As a quotient of a totally disconnected, compactly generated group of polynomial growth, $H / C$ is then a discrete, finitely generated group of polynomial growth.

By Gromov's theorem on finitely generated groups of polynomial growth (that is, the Main Theorem in [Gro81] or Corollary 1.6 in [Kle07]) $H / C$ is virtually nilpotent. Let $H_{0}$ be the inverse image of a nilpotent subgroup, $N$ say, of finite index in $H / C$ under the canonical projection $H \rightarrow H / C$, let $\mathscr{H}_{0}$ be the inverse image of $H_{0} \cap \mathscr{H}$ under the map $\mathscr{H} \rightarrow H$ and let $\mathcal{N}_{0}$ be the kernel of the composite map $\mathscr{H}_{0} \hookrightarrow \mathscr{H} \rightarrow H \rightarrow H / C$.

Then $\mathscr{H}_{0}$ has finite index in $\mathscr{H}, \mathcal{N}_{0}$ is normal in $\mathscr{H}_{0}$ and $\mathscr{H}_{0} / \mathcal{N}_{0}$ is a subgroup of the finitely generated, nilpotent group $N$. Every subgroup of a finitely generated nilpotent group is finitely generated; see e.g. [Bro73], Lemma 2. Therefore $\mathscr{H}_{0} / \mathcal{N}_{0}$ is a finitely generated nilpotent group.

The proof of Theorem 2.1 will therefore be complete, once we show that there exists $V \in \mathscr{B}(G)$ such that $\mathcal{N}_{0}$ stabilizes $V$. To see this, we use that the group of automorphisms of the graph $X$ induced by the subgroup $\mathcal{N}_{0}$ of $\mathscr{H}$ is contained in $C$ by the definition of $\mathcal{N}_{0}$. Since the group $C$ is compact, the set of images of the vertex $O \in X$ under $C$ is finite. The same conclusion can be drawn for the subgroup $\mathcal{N}_{0}$ of $C$. Therefore the subgroup $V:=\bigcap_{n_{0} \in \mathcal{N}_{0}} n_{0}(O)$ is a compact, open subgroup of $G$. By the definition of $V$, the group $\mathcal{N}_{0}$ stabilizes $V$, and our proof is complete. 
Proof of Theorem 2.3. As already noted in Section 2, modulo the claim contained therein on the rank of $\mathscr{H}_{0}$, Theorem 2.3 follows from Theorems 2.1, using Theorem 2.2 .

To determine the rank of the subgroup $\mathscr{H}_{0}$ of $\mathscr{H}$ we argue as follows. Applying Lemma 1.2 with $\boldsymbol{B}:=\mathscr{B}(G)$ and the natural action of $\mathscr{H}$ on $\boldsymbol{B}$ we see that all orbits of $\mathscr{H}$ in $\mathscr{B}(G)$ have the same type of growth, which, by assumption, is polynomial of degree $n$ but not of degree $n-1$. Since $\mathscr{H}_{0}$ has finite index in $\mathscr{H}$, the type of growth of the orbits of $\mathscr{H}_{0}$ in $\mathscr{B}(G)$ is the same as the type of growth of the orbits of $\mathscr{H}$ in $\mathscr{B}(G)$. If we choose a minimizing subgroup, $V$ say, for the flat group $\mathscr{H}_{0}$, whose rank is $r$ say, then Theorem 1.1 implies that the metric space $\mathscr{H}_{0} . V$ has polynomial growth of degree $r$ but not of degree $r-1$. We conclude that $r$ equals $n$ as claimed. This concludes the proof of Theorem 2.3.

\section{Subgroup of bounded conjugacy classes and the proof of Theorem 2.5}

As already noticed in Section 2, the proof of the existence of the flat subgroup $\mathscr{H}_{0}$ in Theorem 2.3 is not constructive. To find $\mathscr{H}_{0}$, we relied on Proposition 1 in [Los01] and on Gromov's Theorem, both of which are non-constructive.

Nevertheless Theorem 2.5 gives a useful characterization of the maximal, normal flat subgroup of a virtually flat group $\mathscr{H}$ of automorphisms and in this section we prove that theorem. The first step towards that result is the following Lemma. Its proof is left to the reader.

Lemma 4.1 (Bornological group structure on the automorphism group). Let $G$ be a totally disconnected, locally compact group and $\mathscr{H}$ a group of automorphisms of $G$. Then the collection of bounded subsets of $\mathcal{H}$ is a bornological group structure on $\mathcal{H}$; that is, singleton sets are bounded, subsets of bounded sets are bounded, finite unions of bounded sets are bounded and products and inverses of bounded sets are bounded.

The following proposition is a consequence of Lemma 4.1; its proof is straightforward and is left to the reader.

Proposition 4.2 (Bounded conjugacy classes form a normal subgroup). Let $G$ be a totally disconnected, locally compact group and $\mathscr{H}$ a group of automorphisms of $G$. Then the set $\mathscr{H}_{\mathrm{FC}_{d}}:=\left\{\varphi \in \mathscr{H}: \varphi^{\mathscr{H}}\right.$ is bounded $\}$ is a normal subgroup of $\mathscr{H}$ and for any such $\mathscr{H}$ we have $\left(\mathscr{H}_{\mathrm{FC}_{d}}\right)_{\mathrm{FC}_{d}}=\mathscr{H}_{\mathrm{FC}_{d}}$.

The next proposition explains why one should expect the subgroup of bounded conjugacy classes of a group of automorphisms to be related to flat subgroups.

Proposition 4.3 (A flat group has bounded conjugacy classes). Suppose that $\mathscr{H}$ is a flat group of automorphisms of a totally disconnected, locally compact group. Then $\mathscr{H}=\mathscr{H}_{\mathrm{FC}_{d}}$. 
Proof. Let $\varphi$ be an arbitrary element of $\mathscr{H}$. Choose a compact, open subgroup $O$ which is minimizing for $\mathscr{H}$. Then, by part 1 of Theorem 1.1, we have

$$
\varphi^{\mathscr{H}} . O=\varphi \cdot\left[\varphi^{-1}, \mathscr{H}\right] . O \subseteq \varphi \cdot \mathscr{H}(1) . O=\varphi \cdot \mathscr{H}_{O} . O=\varphi(O),
$$

and hence $\varphi^{\mathscr{H}}$ is bounded.

We now turn to the proof of Theorem 2.5. That proof will consist of two steps; (Step 1) under the assumptions of Theorem 2.5 it will be shown in Lemma 4.4 that $\mathscr{H}_{\mathrm{FC}_{d}}$ is a normal subgroup of $\mathscr{H}$ that contains every flat subgroup of finite index;

(Step 2) Lemma 4.5 applied with $\mathscr{H}_{\mathrm{fcd}}:=\mathscr{H}_{\mathrm{FC}_{d}}$ shows that $\mathscr{H}_{\mathrm{FC}_{d}}$ is flat.

Lemma 4.4. Suppose that $\mathscr{H}$ is a group of automorphisms of a totally disconnected, locally compact group that is virtually flat. Then $\mathscr{H}_{\mathrm{FC}_{d}}$ is a normal subgroup of $\mathcal{H}$ that contains every flat subgroup of finite index. In particular, $\mathcal{H}_{\mathrm{FC}_{d}}$ has finite index in $\mathscr{H}$.

Proof. Taking Proposition 4.2 into account, we only need to show that $\mathscr{H}_{\mathrm{FC}_{d}}$ contains every flat subgroup of finite index. Let $\mathscr{H}_{0}$ be one such subgroup of $\mathscr{H}$ and $\varphi_{0}$ an arbitrary element of $\mathscr{H}_{0}$. The argument of Proposition 4.3 shows that the set $\varphi_{0}^{\mathscr{H}_{0}}$ is a bounded subset of $\mathscr{H}_{0}$ and hence of $\mathscr{H}$. Write $\mathscr{H}$ as $\bigcup_{i=1}^{k} \alpha_{i} \mathscr{H}_{0}$. Then $\varphi_{0}^{\mathscr{H}}=\bigcup_{i=1}^{k} \alpha_{i} \varphi_{0}^{\mathscr{H}_{0}} \alpha_{i}^{-1}$ is a finite union of bounded subsets of $\mathscr{H}$, and hence is bounded. We conclude that $\varphi_{0}$ is contained in $\mathscr{H}_{\mathrm{FC}_{d}}$, which proves the claim.

Lemma 4.5. Let $G$ be a totally disconnected, locally compact group and $\mathscr{H}_{\mathrm{fcd}} \leqslant$ $\operatorname{Aut}(G)$. Assume that there is an $O$ in $\mathcal{B}(G)$ such that the orbit $\mathscr{H}_{\mathrm{fcd}} . O$ is proper and coarsely connected (this holds e.g. if $\mathscr{H}_{\mathrm{fcd}}$ is virtually flat of finite rank). Assume further that $\mathscr{H}_{\mathrm{fcd}}=\left(\mathscr{H}_{\mathrm{fcd}}\right)_{\mathrm{FC}_{d}}$. Then there is $\mathcal{N}_{0} \unlhd \mathscr{H}_{\mathrm{fcd}}$ and $V \in \mathscr{B}(G)$ such that $\mathcal{N}_{0}$ stabilizes $V$ and $\mathscr{H}_{\mathrm{fcd}} / \mathcal{N}_{0}$ is a finitely generated abelian group. In particular, $\mathscr{H}_{\mathrm{fcd}}$ is flat of finite rank.

Proof. We first verify that the condition that $\mathscr{H}_{\text {fcd }}$ is virtually flat of finite rank implies that there is an $O$ in $\mathscr{B}(G)$ such that the orbit $\mathscr{H}_{\mathrm{fcd}} . O$ is proper and coarsely connected. Let $\mathscr{H}_{f}$ be a flat subgroup of finite index in $\mathscr{H}_{\mathrm{fcd}}$ and $O$ a minimizing subgroup for $\mathscr{H}_{f}$. By the remark in the paragraph preceding Theorem 2.1, the orbit $\mathscr{H}_{f} . O$ is proper and coarsely connected. Since $\mathscr{H}_{f}$ has finite index in $\mathscr{H}_{\text {fcd }}$, the analogous statement holds for the orbit $\mathscr{H}_{\mathrm{fcd}} . O$.

Define a graph $X$ as in the proof of Theorem 2.1. Recall that an element whose conjugacy class has compact closure is called an $\overline{\mathrm{FC}}$-element, while a group consisting of $\overline{\mathrm{FC}}$-elements is called an $\overline{\mathrm{FC}}$-group. Denote by $H$ the subgroup of $\overline{\mathrm{FC}}$-elements in the closure, $\overline{\mathscr{H}_{\text {fcd }}}$, of the group of automorphisms of $X$ induced by $\mathscr{H}_{\text {fcd }}$ in the group of all graph-automorphisms of $X$. As in the proof of Theorem 2.1 we conclude that the group $\overline{\mathscr{H}_{\text {fcd }}}$ is totally disconnected and locally compact. Theorem 2 in [Möl03] implies that $H$ is a closed subgroup of $\overline{\mathscr{H}_{\mathrm{fcd}}}$. Note that by our assumption $\mathscr{H}_{\mathrm{fcd}}$ equals 
$\left(\mathscr{H}_{\mathrm{fcd}}\right)_{\mathrm{FC}_{d}}$ and by the definition of the topology on $\operatorname{Aut}(X), H$ contains the image of $\mathscr{H}_{\mathrm{fcd}}$ in $\operatorname{Aut}(X)$ and hence equals $\overline{\mathscr{H}_{\mathrm{fcd}}}$. Thus $H$ is a totally disconnected, locally compact, compactly generated $\overline{\mathrm{FC}}$-group.

Using Theorem 3.20 in [GM71], and remembering that $H$ is totally disconnected, we conclude that the group $H$ has a compact, normal subgroup $P$ with discrete, torsion free, finitely generated abelian quotient $A$.

Put $\mathscr{H}_{0}:=\mathscr{H}_{\text {fcd }}$ and let $\mathcal{N}_{0}$ be equal to the kernel of the composite homomorphism $\mathscr{H}_{\mathrm{fcd}} \rightarrow H \rightarrow A$. Then $\mathcal{N}_{0}$ is a normal subgroup of $\mathscr{H}_{0}$ and $\mathscr{H}_{0} / \mathcal{N}_{0}$ is a finitely generated, nilpotent (in fact, abelian) group.

Using that $\mathcal{N}_{0}$ is contained in the compact subgroup $P$ of $\operatorname{Aut}(X)$ we conclude as in the proof of Theorem 2.1 that there exists $V \in \mathcal{B}(G)$ such that $\mathcal{N}_{0}$ stabilizes $V$. Applying Theorem 2.2 we conclude that the group $\mathscr{H}_{0}=\mathscr{H}_{\mathrm{fcd}}$ is flat of finite rank. We have shown all parts of our claim.

As explained in the paragraph preceding Lemma 4.4, Theorem 2.5 follows from what we have just shown.

From Lemmas 4.4 and 4.5 we now obtain, in the 'proper case', another characterization of virtually flat groups of automorphisms in terms of their elements with bounded conjugacy class.

Theorem 4.6. Let $G$ be a totally disconnected, locally compact group and let $\mathscr{H}$ be a group of automorphisms of $G$. Assume that there is an $O$ in $\mathcal{B}(G)$ such that $\mathscr{H} . O$ is proper and coarsely connected. Then the following conditions are equivalent:

(1) $\mathscr{H}$ is virtually flat of finite rank.

(2) $\left|\mathscr{H}: \mathscr{H}_{\mathrm{FC}_{d}}\right|$ is finite.

Proof. That (1) implies (2) follows from Lemma 4.4. Assume conversely that (2)

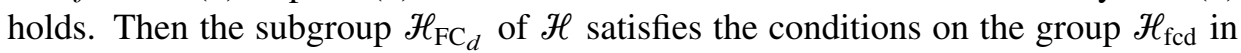
Lemma 4.5. Applying Lemma 4.5 we conclude that the group $\mathscr{H}_{\mathrm{FC}_{d}}$ is flat of finite rank. Since $\mathscr{H}_{\mathrm{FC}_{d}}$ is a subgroup of finite index in $\mathscr{H}$ by assumption, $\mathscr{H}$ is virtually flat of finite rank, which is the content of statement (1).

\section{An example and an application}

A group of automorphisms of a totally disconnected, locally compact group whose orbits in the space of compact, open subgroups are quasi-flats need not be a flat group as the following example illustrates. Conjecture 16 in the paper [BRW07] is therefore false as stated; the first and third author of the current paper should have known this while being involved in writing the paper [BRW07], because they obtained Theorem 5.3 below, which also illustrates this point, by different methods in unpublished work done in the year 2000. 
Example 5.1. Let $p$ be a prime number and $G$ the additive group of the 2-dimensional vector space over $\mathbb{Q}_{p}$. The metric space of compact, open subgroups of $G$ is proper. Let $\mathscr{H}$ be the group of automorphisms of $G$ that is generated by the following two linear transformations:

$$
s_{1}:=\left(\begin{array}{cc}
0 & 1 \\
1 & 0
\end{array}\right), \quad s_{2}:=\left(\begin{array}{cc}
0 & p^{-1} \\
p & 0
\end{array}\right)=\left(\begin{array}{ll}
0 & 1 \\
1 & 0
\end{array}\right) \cdot\left(\begin{array}{cc}
p & 0 \\
0 & p^{-1}
\end{array}\right)=: s_{1} u .
$$

We will show that the orbits of compact, open subgroups under $\mathscr{H}$ are quasi-isometric to $\mathbb{R}$, but that the group $\mathcal{H}$ is not flat.

Proof. The group $\mathscr{H}$ is isomorphic to the infinite dihedral group with canonical generators $s_{1}$ and $s_{2}$.

The group $\mathcal{T}:=\langle u\rangle$ has index 2 in $\mathscr{H}$. Since $\mathcal{T}$ is generated by one element, it is a flat group whose rank is either 0 or 1 ; this may be seen for example by applying Lemma 2 in [BW06] with $u:=\alpha$. The group $\mathcal{T}$ has unbounded orbits in $\mathscr{B}(G)$; hence the rank of $\mathcal{T}$ must be larger than 0 and hence is 1 . Using Theorem 1.1 we conclude the orbits of the group $\mathcal{T}$ in $\mathscr{B}(G)$ are quasi-isometric to $\mathbb{R}$. Since $\mathcal{T}$ has finite index in $\mathscr{H}$ the same is true of the orbits of the group $\mathscr{H}$ in $\mathscr{B}(G)$, as claimed.

We now show by contradiction that the group $\mathscr{H}$ is not flat. For suppose it were. Then the elements $s_{1}$ and $s_{2}$ are contained in the subgroup $\mathscr{H}(1)$, because they have finite order. Since $s_{1}$ and $s_{2}$ generate $\mathscr{H}$, we then must have $\mathscr{H}=\mathscr{H}(1)$, the rank of $\mathscr{H}$ would be 0 and all orbits of $\mathscr{H}$ in $\mathcal{B}(G)$ would be bounded. This is a contradiction to what we have shown to be the case above and we conclude that $\mathscr{H}$ is not flat, as claimed.

Remark 5.2. The phenomenon illustrated in the above example does not occur with groups of automorphisms whose orbits in $\mathcal{B}(G)$ are bounded. Under this condition one need not even impose a condition on $\mathscr{B}(G)$. That is, any group of automorphisms with bounded orbits in $\mathscr{B}(G)$ is flat of rank 0 . This statement is Proposition 5 in [BW06]. It is a consequence of the main result of Schlichting's paper [Sch80]; the latter result has been generalized in [BL89] and [Wag98].

This stronger form of the geometric flatness criterion in the rank-0-case has played an important role in the recent study, by Y. Shalom and G. Willis, of almost normal subgroups of arithmetic groups via suitable completions.

We now show how the results of this paper lead to a remarkably simple and straightforward proof of the following result, which again illustrates the problem of using virtual notions when dealing with flatness. The following notions are used: A flat subgroup is a subgroup that is flat as a group of inner automorphisms; the flat rank of a totally disconnected, locally compact group is the supremum of the ranks of its flat subgroups. 
Theorem 5.3. Let $k$ be a nonarchimedean local field and $\boldsymbol{G}$ a connected semisimple group that is defined over $k$. Then for any maximal $k$-split torus $\boldsymbol{S}$ of $\boldsymbol{G}$ the group $Z_{\boldsymbol{G}}(\boldsymbol{S})(k)$ is a maximal flat subgroup of $\boldsymbol{G}(k)$ of maximal rank, equal to the $k$-rank of $\boldsymbol{G}$. In particular, the group $\mathcal{N}_{\boldsymbol{G}}(\boldsymbol{S})(k)$ is not flat; however the orbits of this group in $\mathscr{B}(\boldsymbol{G}(k))$ are quasi-flats of dimension $k-\operatorname{rank}(\boldsymbol{G})=$ flat-rank $(\boldsymbol{G}(k))$.

Proof. It will be obvious from the proof, that an analogous result may be obtained in other cases also. The action of the group $\boldsymbol{G}(k)$ on the Bruhat-Tits building $X(\boldsymbol{G}, k)$ of $\boldsymbol{G}$ over $k$ will be used.

Let $\boldsymbol{S}$ be a maximal $k$-split torus of $\boldsymbol{G}$. The apartment $A_{\boldsymbol{S}}$ associated to $\boldsymbol{S}$ is an affine subspace of $X(\boldsymbol{G}, k)$ of maximal dimension. The group $N:=\mathcal{N}_{\boldsymbol{G}}(\boldsymbol{S})(k)$ stabilizes $A_{S}$ and acts there by affine isometries. The subgroup of $N$ that acts by translations on $A_{\boldsymbol{S}}$ is $T:=\mathfrak{Z}_{\boldsymbol{G}}(\boldsymbol{S})(k)$. Since $X(\boldsymbol{G}, k)$ is also the building defined by a saturated $B N$-pair (namely $(B, N)$, where $B$ is a suitable Iwahori subgroup) the group $N=\mathcal{N}_{\boldsymbol{G}}(\boldsymbol{S})(k)$ is the full stabilizer of $A_{\boldsymbol{S}}$ in $\boldsymbol{G}(k)$.

We next use Theorem 7 from [BRW07] to turn the statements of the previous paragraph into statements about the action of $\boldsymbol{G}(k)$ on $\mathcal{B}(\boldsymbol{G}(k))$. We conclude that the orbits of $N$ and $T$ in $\mathscr{B}(\boldsymbol{G}(k))$ are quasi-flats of dimension $k-\operatorname{rank}(\boldsymbol{G})$. That $k-\operatorname{rank}(\boldsymbol{G})=$ flat-rank $(\boldsymbol{G}(k))$ follows from Corollary 19 in [BRW07].

The orbits of the stabilizer of a chamber in $A_{\boldsymbol{S}}$ under $N$ and $T$ are proper; in fact, the metric space $\mathcal{B}(\boldsymbol{G}(k))$ is proper. From either Theorem 2.3 or Theorem 1.4 we infer that $N$ and $T$ are virtually flat subgroups of $\boldsymbol{G}(k)$ of maximal rank. We claim next that the subgroup of bounded conjugacy classes $N_{\mathrm{FC}_{d}}$ of $N$ equals $T$. Applying Theorem 2.5 to this statement yields that $T$ is flat; since we know that $N$ is the full stabilizer of $A_{\boldsymbol{S}}$, every flat subgroup containing $T$ must be contained in $N$ and we will obtain our remaining claims.

We are therefore reduced to proving that $N_{\mathrm{FC}_{d}}=T$. The group $T$ is contained in $N_{\mathrm{FC}_{d}}$ because it acts by translations on $A_{S}$ and any conjugate of a translation is a translation which displaces points by the same amount as the original translation. The set $N \backslash T$ consists of elements which act as reflections or rotations on $A_{S}$; no such element can be contained in $N_{\mathrm{FC}_{d}}$, because given a fixed point of $A_{S}$ that point will be displaced by an arbitrarily large amount by a conjugate of such a transformation by a translation of sufficiently large displacement. Thus $N_{\mathrm{FC}_{d}}=T$, concluding our proof.

It is uncertain whether one can also find a minimizing subgroup for the group $Z_{\boldsymbol{G}}(\boldsymbol{S})(k)$ in the previous result along the same lines. After all, our approach avoided the task of finding a minimizing subgroup.

A minimizing subgroup for the group $\mathcal{Z}_{\boldsymbol{G}}(\boldsymbol{S})(k)$ is known; compare Theorem 5.4 below. We omit the proof of this result, hoping to find a simpler approach to it which might also apply in more general situations.

Theorem 5.4. Let $k$ be a nonarchimedean local field and $\boldsymbol{G}$ a connected semisimple group that is defined over $k$. Then for any maximal $k$-split torus $\boldsymbol{S}$ of $\boldsymbol{G}$ the stabilizer 
of any chamber of the affine apartment corresponding to $\boldsymbol{S}$ is minimizing for the group $\mathrm{Z}_{\boldsymbol{G}}(\boldsymbol{S})(k)$.

\section{References}

[Aus65] L. Auslander, An account of the theory of crystallographic groups. Proc. Amer. Math. Soc. 16 (1965), 1230-1236. Zbl 0133.28605 MR 0185012

[Bau07] U. Baumgartner, Totally disconnected, locally compact groups as geometric objects: a survey of work in progress. In Geometric group theory, Geneva and Barcelona Conferences, Trends Math., Birkhäuser Verlag, Basel 2007, 1-20. Zbl 1162.22001 MR 2395785

[BRW07] U. Baumgartner, B. Rémy, and G. A. Willis, Flat rank of automorphism groups of buildings. Transform. Groups 12 (2007), 413-436. Zbl 1145.22012 MR 2356316

[BW06] U. Baumgartner and G. A. Willis, The direction of an automorphism of a totally disconnected locally compact group. Math. Z. 252 (2006), 393-428. Zbl 1085.22002 MR 2207803

[BL89] G. M. Bergman and H. W. Lenstra, Jr., Subgroups close to normal subgroups. J. Algebra 127 (1989), 80-97. Zbl 0641.20023 MR 1029404

[Bro73] I. D. Brown, Representation of finitely generated nilpotent groups. Pacific J. Math. 45 (1973), 13-26. Zbl 0243.22007 MR 0352324

[dCTV07] Y. de Cornulier, R. Tessera, and A. Valette, Isometric group actions on Hilbert spaces: growth of cocycles. Geom. Funct. Anal. 17 (2007), 770-792. Zbl 1129.22004 MR 2346274

[GdlH90] É. Ghys and P. de la Harpe (eds.), Sur les groupes hyperboliques d'après Mikhael Gromov. Progr. Math. 83, Birkhäuser, Boston 1990. Zbl 0731.20025 MR 1086648

[Gro81] M. Gromov, Groups of polynomial growth and expanding maps. Inst. Hautes Études Sci. Publ. Math. (1981), 53-73. Zbl 0474.20018 MR 623534

[GM71] Siegfried Grosser and Martin Moskowitz, Compactness conditions in topological groups. J. Reine Angew. Math. 246 (1971), 1-40. Zbl 0219.22011 MR 0284541

[IS91] W. Imrich and N. Seifter, A survey on graphs with polynomial growth. Discrete Math. 95 (1991), 101-117. Zbl 0761.05048 MR 1141934

[Kle07] B. Kleiner, A new proof of Gromov's theorem on groups of polynomial growth. Preprint 2007. arXiv:0710.4593

[Los01] V. Losert, On the structure of groups with polynomial growth. II. J. London Math. Soc. (2) 63 (2001), 640-654. Zbl 1010.22008 MR 1825980

[LS01] A. Lukács and N. Seifter, Finite contractions of graphs with polynomial growth. European J. Combin. 22 (2001), 85-90. Zbl 0964.05028 MR 1808087

[Mö103] R. G. Möller, $\mathrm{FC}^{-}$-elements in totally disconnected groups and automorphisms of infinite graphs. Math. Scand. 92 (2003) , 261-268. Zbl 1132.22002 MR 1973946

[Sch80] G. Schlichting, Operationen mit periodischen Stabilisatoren. Arch. Math. (Basel) 34 (1980), 97-99. Zbl 0449.20004 MR 583752 
[Sei91] N. Seifter, Groups acting on graphs with polynomial growth. Discrete Math. 89 (1991), 269-280. Zbl 0739.05041 MR 1112445

[Sha04] Y. Shalom, Harmonic analysis, cohomology, and the large-scale geometry of amenable groups. Acta Math. 192 (2004), 119-185. Zbl 1064.43004 MR 2096453

[SW07] Y. Shalom and G. A. Willis, Commensurated subgroups of arithmetic groups, totally disconnected groups and adelic rigidity. Preprint 2009. arXiv:0911.1966

[Tro84] V. I. Trofimov, Graphs with polynomial growth. Mat. Sb. (N.S.) 123 (165) (1984), 407-421; English transl. Math. USSR-Sb. 51 (1985), 405-417. Zbl 0548.05033 MR 735714

[Wag98] F. O. Wagner, Almost invariant families. Bull. London Math. Soc. 30 (1998), 235240. Zbl 0921.03056 MR 1608090

[Wil04] G. A. Willis, Tidy subgroups for commuting automorphisms of totally disconnected locally compact groups: An analogue of simultanous triangularisation of matrices. New York J. Math 10 (2004), 1-35. Zbl 1029.22006 MR 2052362

Received July 31, 2008; revised January 23, 2009

U. Baumgartner, School of Mathematical and Physical Sciences, The University of Newcastle, University Drive, Building V, Callaghan, NSW 2308, Australia

E-mail: Udo.Baumgartner@newcastle.edu.au

G. Schlichting, Technische Universität München, Center of Mathematical Sciences, M8, 85748 Garching bei München, Germany

E-mail: schlicht@mathematik.tu-muenchen.de

G. Willis, School of Mathematical and Physical Sciences, The University of Newcastle, University Drive, Building V, Callaghan, NSW 2308, Australia

E-mail: George.Willis@newcastle.edu.au 